\title{
A Fabric Defect Classification Based on Two-dimensional Sparse Representations and a Norm Optimization
}

\author{
Yuanshao Hou ${ }^{1, a}$ and Jiande Fan ${ }^{1, b}$ \\ 'Zhengzhou University of Industrial Technology, Information Engineering, Zhengzhou, China, 451150 \\ a516063795@qq.com, b568498042@qq.com
}

Keywords: Two-dimensional sparse; Fabric defects; Norm optimization; Classification

\begin{abstract}
Sampling loss of the structural information of the image for the one-dimensional compression and bring about the loss of recognition accuracy, we propose the concept of two-dimensional compression samples. Using a set of sparse-based perception to get the sparse data on the raw data of the defect, fabric defect two-dimensional sparse. Finally, use of norm optimization method accurately decrypt the compressed data, the eigenvalues of different fabric defect classification. This approach solves the proliferation of data collection and the sensor waste greatly reduces the computational complexity, fabric defect classification, and thus to lay a theoretical foundation for machine vision to identify fabric defects.
\end{abstract}

Introduction

Fabric defect is not only affects the quality of cloth but also affects the prices of textiles. As cord fabric is a special kind of textile products, its production speed is fast, wide, surface texture is relatively complex, and has a great large of defect types. Therefore, how to made rapid detection under complicated background in cord fabric defect puts forward higher requirements on detection and recognition technology. With the continuous development of image acquisition technology, fabric image collection has been solved well.so, to find effective defect detection identification algorithms become a research focus. However, with the improvement of the data acquisition device and sensor data processing ability, increasing the resolution of the image. Image recognition has higher dimension of data to be processed, and the data dimension of ascension makes complete real-time image processing and recognition is becoming more and more difficult. In order to solve the problem of data dimension raise and improve the speed of image recognition, we need find an effective way to reduce the data redundancy and complete raw data dimension reduction. In addition, the dimension reduction of image process is not only to reduce the dimension of data but also must seize the image of the main information and ignore the secondary information, so it should also is a kind of effective means of feature extraction. In this paper, two-dimensional compression sampling method is proposed on the basis of the one-dimensional compression sampling.

\section{Two-dimensional Sparse Representation Theory}

The research of Image analysis indicates that image recognition based on machine vision can be summed up in two-step processes, the first step is extracting the feature and the second is classification process. So the fabric defect detection and recognition is essentially a kind of image feature extraction and then classification problems. And sparse representation based on norm optimization theory in recent years has brought us new ideas. If, according to the theory of vector under some kind of transformation is sparse (i.e., vector zero elements of the majority), then you can use this kind of sparse underdetermined random linear mapping of the vector, and don't have to worry about losing any information. As long as the mapping matrix satisfy certain conditions, we can use norm optimization technique by these" under sampling value" (sampling frequency is lower than the classic Nyquist 
frequency) accurately reconstruct the original vector.

So far, the traditional habit of the study of image feature extraction and classification algorithm is usually from the perspective of the vector (1d), and the processing method of one dimension does not take into account the image matrix structure, which usually leads to higher computational overhead and identification of instability.

The Meaning of the Two-dimensional Sparse Representation. We always consider that the traditional one-dimensional feature representation and pattern classification directly used for image recognition will lead to the following drawbacks: (1) the two-dimensional structure of image information lost, and bring losses to the identification accuracy under the same conditions. (2) The traditional algorithm transform the data into vector form first, and this kind of "vectorization method will result in an increase in computational complexity in the process of sampling and reconstruction. For the above shortcomings, the use of image matrix structure (2d) can complete the expansion of the one-dimensional method to two-dimensional. Expanded form of $2 \mathrm{~d}$ image representation and classifier to a great extent solving the shortcomings of the one-dimensional algorithm, and can drive the upgrading and transformation of one dimensional image recognition algorithm, then fabric defect detection and recognition could have a positive impact.

One-dimensional Sampling to Two-dimensional Sparse Sampling. Before the introduction of two dimensional compression sampling, we first do a simple review for one-dimensional compression sampling concepts: It is assumed that $\mathrm{x}$ is an image which $\mathrm{x}$ is a $\mathrm{M} \times$ Nmatrix, we transform matrix $\mathrm{x}$ to vector ${ }^{(v e c)} \in R^{M \times N}$, so we can get the vector feature of one-dimensional image compress form by the Eq (1).

$$
y^{(v e c)}=\Phi x^{(v e c)}
$$

Which $\Phi \in R^{M(v e c) \times(M N)}\left(M^{(v e c)}<M N\right)$ is underdetermined random matrix (Random distribution option Gaussian distribution) that meet the conditions of RIP.

If we don't vectoring $\mathrm{x}$ in our study, keeping $x \in R^{M \times N}$ and making Eq (1) a little change, then we can get a two- dimension compress random projection $\mathrm{Eq}(2)$ :

$$
y=\Phi_{R} x \Phi_{c}
$$

Which $\Phi_{R} \in R^{m \times M} \Phi_{c} \in R^{n \times N}(m<M, n<N)$ are Line sampling matrix (row map) and sampling matrix column (column map). $\Phi_{R}$ and $\Phi_{C}$ are underdetermined random matrix that Meet the conditions of RIP.what different with one-dimension compress sampling is y from $\mathrm{Eq}(2)$ is not a vector but a matrix $\left(y \in R^{M \times N}\right)$.we called matrix y two-dimension compress sampling mesure.

It is assumed that $\Phi_{R}^{(i)}$ is $\mathrm{i}$-th $(i=1, \ldots, m)$ vector and $\Phi_{c}^{(j)}$ is $\mathrm{j}$-th $(j=1, \ldots, n)$ vector in matrix $\Phi_{R}$. It is not difficult to find that:

$$
y^{(i, j)}=\Phi_{R}^{(i)} x \Phi_{c}^{(j)}
$$

Eq (3) shows the matrix mapping of each element contains a row and column mapping part of extracting information from the matrix $\mathrm{x}$.

It can preliminarily prove oretically and experimentally, as long as it is piecewise smooth (can be sparse through a transformation), or $x$ itself a sparse matrix, and the number of rows/columns of the matrix is greater than a certain threshold (but less than the number of row/column of $x$ ). The amount of information contained in $\mathrm{y}$ and as much as $\mathrm{x}$ has, so you can use the technology of convexification reconstruction from $\mathrm{y}$ to $\mathrm{x}$.

In practical application, if the acquisition image $\mathrm{f}$ itself is not sparse but on a $\psi$ base of transform coefficient $\theta$ is sparse or compressible, namely $f=\psi \theta$. Then it is said image $\mathrm{f}$ can be compressed. 
Sparse representation theory not only can be used in the feature representation, but also can be used for the design of classifier. In image recognition, for example, assume that the input image can be expressed as linear and sample training set, and the linear training set's coefficient vector is sparse, and by the position of the sparse vector of non-zero value can classify the input image. And the process is actually based on sparse vector norm optimization reconstruction process is based on 11 norm optimization reconstruction process $[1,2]$.

The Representation of Two-dimensional Sparse Training Set. Two-dimensional sparse representation of ideas can also be represented in figure 1: figure 1 dictionary $A$ is underdetermined matrix that constructed of the training set samples, and each column is the known ID mode vector, $y$ is the input mode vector. When the sample is fully enough in the dictionary $A, \mathrm{y}$ can be expressed as the linear of related patterns in $A$. The coefficient vector $\alpha$ shall be sparse, so the non-zero value in $\alpha$ can locate the relevance vector included in $A$. we can determined that if the relevant samples belong to the first class on the basis of this classification, so if the relevance sample belongs to the $\mathrm{i}$-th class of $A$ then the image should be classified i-th class. Mathematically to the above ideas can also be for the following:

$$
y=\alpha_{i, 1} v_{i, 1}+\alpha_{i, 2} v_{i, 2}+\ldots+\alpha_{i, n_{i}} v_{i, n_{i}}=A_{i} \alpha_{i}
$$

$A_{i}=\left[u_{i, 1}, u_{i, 2}, \ldots, u_{i, n}\right]$ is i-th training sample set, $\alpha_{i}=\left[\alpha_{i, 1} \alpha_{i, 2}, \ldots, \alpha_{i, n}\right]^{T}$ is coefficients vectors Eq [3]. For all the training set of sample (totally k class), the above formula can also be written as the following form:

$$
y=\left[A_{1}, A_{2}, \ldots, A_{k}\right]\left[\alpha_{1}, \alpha_{2}, \ldots, \alpha_{k}\right]^{T}=A \alpha
$$

$A=\left[A_{1}, A_{2}, \ldots, A_{k}\right]$ is total training set; $\alpha=\left[\alpha_{1}, \alpha_{2}, \ldots, \alpha_{k}\right]^{T}$ is coefficients vectors ; as $\alpha$ is related with $A_{i}$ only, so $\alpha$ must be sparse vector and the location and size of sparse vector is Closely linked $A_{i}$.we can confirm ID by sloving $\alpha$.

Because $\mathrm{A}$ is an underdetermined matrix, so Eq (5) has an infinite number of solutions. The above problem can be transformed to a convex optimization problem by sloving minimize 11 norm min $\|\alpha\|$, then got the sparse solution of $\alpha$ These ideas have been sums up by American mathematician D. Donoho "for large systems of equations, the minimum 11norm solution is also the minimum 10 norm solution "[3].

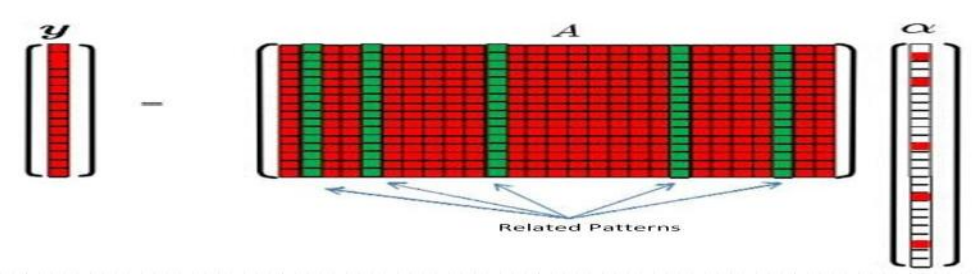

Figure 1. Pattern recognition method based on sparse representation

Two-dimensional Sparse Representation of the Constraint Condition. By shannon sampling theorem, the sampling frequency is not only less than the Nyquist frequency (the spectrum of analog signal) twice of the highest frequency can restore the original signal distortion. However, Shannon's sampling theorem, the signal is assumed in addition to using limited bandwidth, without the use of any other a priori information, the collected data is in large redundancy.

Sparse representation of the two-dimensional data acquisition is not directly, but through a specific set of waveform data to perceive that, in order to accurately reconstruct the original data set we must be 
used to sense particular waveform data, and make use of the original waveform to measure the specific degree of coherence of data, only when the degree of coherence in the acceptable range to optimize the method using norm accurate reconstruction of data; the key factors involved in that with a given waveform to the waveform can be used to compress the raw data are irrelevant and the higher the irrelevant is, the greater amount of information the awareness data contained, then get an accurate perception of the reconstruction, the less the amount of data required for the original data ${ }^{[4]}$.

Restricted equidistance principle, consistent with the uncertainty principle and the principle of accurate reconstruction principle those raised abroad principle further answered how to easily extract the signal or image or other useful information from the compressed data ${ }^{[5]}$.

Fig. 2 is a schematic diagram of the sampling process, where in the signal $f$ to be collected only at time zero of $k$ ( $k$ is the degree of sparse) and the k-th non-zero only in this time to effectively capture the signal $f$ of the information, and projected onto a given set of sensing waveforms $\Phi$ (it can be said with a given waveform to perceived $f$ ) to obtain a set of data is much smaller than the original length measurement data $y \quad[6]$.
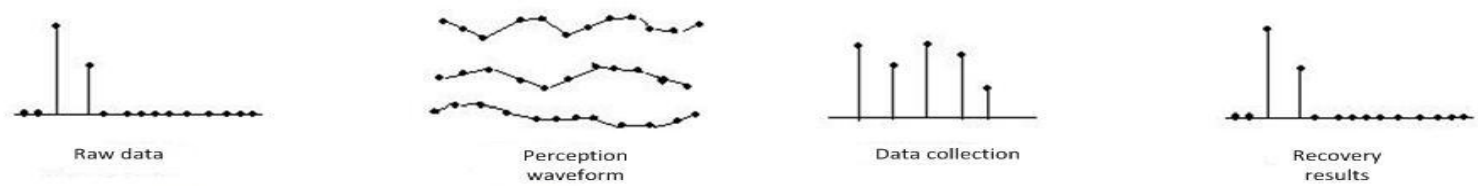

Figure 2. Compressed sensing data collection methods

$$
f \times\left[\phi_{1}, \phi_{2}, \ldots, \phi_{m}\right]^{T}=y \stackrel{l_{1} \text { 最小化 }}{\longrightarrow} \hat{f}
$$

Coherence and Sparsity. $u$ define the degree of coherence between the base and the base is:

$$
u(\phi, \psi)=\max _{1 \leq k, j \leq n}\left|\left(\phi_{k}, \psi_{j}\right)\right|=\max _{1 \leq k, j \leq n}|A(k, j)|
$$

Which $\phi_{k}, \psi_{j}$ are row and column vectors of orthogonal matrix $\phi 、 \psi(k, j$ respectively rows and columns of the matrix). Through knowledge of linear algebra we can get $u(\phi, \psi) \in[1 / \sqrt{n}, 1]$. Therefore, when the measurement matrix is a unit matrix, the maximum degree of coherence of $u$ is 1 . In this case, only the amount of data collected and raw sensory data corresponding to the data to estimate the compressibility of the important information. Therefore, when the degree of coherence between the sensing matrix and the transformation matrix is 1 , only can we using conventional sampling methods. In fact, the most matrixes are substantially incoherent, i.e. the degree of coherence is not 1 .

The minimum degree of coherence matrix norms is formed by the Fourier transform matrix and impulse $\operatorname{Eq} \phi_{k}=\delta\left(t-t_{k}\right)$.and the value is $1 / \sqrt{n}$.he degree of coherence between the Pulse Eq $\phi_{k}=\delta\left(t-t_{k}\right)$ and sine curve is also $1 / \sqrt{n}$.

Data Compressibility. The compressibility of data is that data can be expressed by fewer coefficients in case of little lost or out of a transform domain data in the field of support is relatively small, the data is compressible. Further, when the data in the spatial domain or the transform domain coefficients are belong to ball $l_{p}(0<p<1)$, and the data is also compressible data [7].

In this case indicates that the transform coefficients data $\theta$ of $f$ determined by the given group $f=\psi \theta$, for any given constant $R(R>0)$ and $P(0<P<1)$ satisfy Eq (8) 


$$
|\theta|_{p}=\left\{\sum_{i=1}^{k}\left|\theta_{i}\right|_{p}\right\}^{1 / p} \leq R
$$

When the coefficient $\theta$ belongs to the ball $l_{p}$, set up $\theta_{k}$ is $k$ larger items in $\theta$, and other items in the resulting vector is set to zero, then

$$
\left|\theta-\theta_{K}\right|_{2} \leq \zeta_{2, P}|\theta|_{P}(K+1)^{1 / 2-1 / P}
$$

Which $\zeta_{2, p}$ is constant only depend on $p \in(0,2)$. So in order to make the estimation error is not greater than $\varepsilon$, only to satisfies $k \geq \varepsilon^{(p-2) / 2 p}$, even the $\mathrm{k}$ coefficient keep the most important information of the data.

\section{Conclusion}

In the present study, we believe that norm optimization based on sparse representation and image recognition algorithms can also to be improved [8]. In sparse transform and image reconstruction process is often mixed with noise interference, how to maximize the removal does not affect the case of image reconstruction noise, and make use of the difference between structure of image and sparse matrix to reconstruction and classified fabric defect image [9]. But also need to be improved in the following areas:

(1) In sparse representation, the low-rank representation and manifold structure is closely related to data sparsity, and if low rank representation means and the manifold structure are introduced to the sparse representation of the image will be expected to get better compression results [10].

(2) In the norm optimization, if we can combine the sparsity of the image itself and priori, then the classification of the reconstructed image will get better recovery results.

\section{Acknowledgement}

National Natural Science Foundation of China (NO. 6115200) based on Two Random mapping and optimization of a norm supervised image classification.

\section{References}

[1] Donoho D, Elad M. Optimal Sparse Representation in General (nonorthogonal) Dictionaries via L1 Minimization [C]. New York: In Proceedings of the National Academy of Sciences, 2003.

[2] Donoho D. For Most Large Underdetermined Systems of Linear Equations the Minimal 1-norm Solution is also the Sparsest Solution Comm [J]. On Pure and Applied Math, 2006,59 (6): 797-829.

[3] Wright J, Yang A, Ganesh A, etal. Robust Face Recognition via Sparse Representation [J].IEEE Transactions on Pattern Analysis and Machine Intelligence, 2009, 31 (2): 210-227.

[4] Dai Q H, Fu C J, Ji X Y. Compressive sensing research [J].Chinese J of Computers, 2011, 34(3): 425-434.

[5] Wright J. Robust Face Recognition via Sparse Representation [J]. IEEE Transactions on Pattern Analysis and Machine Intelligence, 2009, 31(2): 227 - 229.

[6] Jiao $1 \mathrm{C}$, Yang S Y, liu F. Development and prospect of compressive sensing [J]. ACTA ELECTRONICA SINICA.2011,31(2):227-229 
[7] Davenport M A. The Smashed Filter for Compressive Classification and Target Recognition[C]. Canada: In Proceedings of Computational Imaging V at SPIE Electronic Imaging, 2007.

[8] Duarte M. Multiscale Random Projections for Compressive Classification [C]. Texas: IEEE on Image Processing (ICIP) 2007.

[9] Wakin M B. Manifold - Based Signal Recovery and Parameter Estimation [J]. Compressive Measurements (Preprint), 2008, 22(3):226-231.

[10]Peyré G. Manifold Models for Signals and Images [J]. Computer Vision and Image Understanding, 2009, 113(2): 249 - 260. 